\title{
Thermal expansion of GaN at low temperatures - a comparison of bulk and homo- and heteroepitaxial layers
}

\author{
Verena Kirchner ${ }^{1}$, Heidrun Heinke ${ }^{1}$, Sven Einfeldt ${ }^{1}$, Detlef Hommel ${ }^{1}$, Jaroslaw Z. \\ Domagala $^{2}$, Michal Leszczynski ${ }^{3}$ \\ ${ }^{1}$ University of Bremen, Institute of Solid State Physics, Bremen, Germany \\ ${ }^{2}$ Polish Academy of Science, Institute of Physics, Dept. of X-ray Studies and Electron \\ Microscopy, Warsaw, Poland \\ ${ }^{3}$ High Pressure Research Center, Unipress, Warsaw, Poland
}

\section{ABSTRACT}

The thermal expansion of different $\mathrm{GaN}$ samples is studied by high-resolution $\mathrm{X}$ ray diffraction within the temperature range of 10 to $600 \mathrm{~K}$. GaN bulk crystals, a homoepitaxial layer and different heteroepitaxial layers grown by metalorganic chemical vapor deposition (MOCVD) and molecular beam epitaxy (MBE) were investigated. Below $100 \mathrm{~K}$ the thermal expansion coefficients (TEC) were found to be nearly zero which has to be taken into account when estimating the thermal strain of GaN layers in optical experiments commonly performed at low temperatures. The homoepitaxial layer and the underlying $\mathrm{GaN}$ substrate with a lattice mismatch of $-6 \cdot 10^{-4}$ showed identical thermal expansion. The comparison between the temperature behavior of lattice parameters of heteroepitaxial layers and bulk $\mathrm{GaN}$ points to a superposition of thermally induced biaxial strain and compressive hydrostatic strain.

\section{INTRODUCTION}

GaN epitaxy is commonly performed on substrates with high mismatch in both lattice parameters and thermal expansion. Whereas the first is relaxed by misfit dislocations within the first few nanometers, the latter results often in thermally induced strain of $\mathrm{GaN}$ epilayers as it is indicated by a variety of optical experiments $[1,2]$. Despite the known importance of thermally induced strain for GaN epitaxy, there are still a lot of open questions and uncertainties related with this subject. On the one hand, this concerns basic material parameters which are essential for a correct description of thermally induced strain in $\mathrm{GaN}$ layers, as the temperature behavior of the TEC of $\mathrm{GaN}$ in the full range from typical growth temperatures down to low temperatures of a few Kelvin as commonly used for optical experiments. Moreover, there were some indications that the TEC could depend on the characteristics of the material as the free electron concentration [3]. On the other hand, the formation of thermally induced strain in $\mathrm{GaN}$ epilayers in dependence on the growth procedures and parameters is far beyond to be fully understood [4].

In the present paper the thermal behavior of differently grown $\mathrm{GaN}$ is studied. This concerns the basic material parameters which could be investigated for bulk and homoepitaxal GaN samples of high crystalline perfection. The thermal expansion of these samples is compared to that of typical heteroepitaxial layers deposited by different growth techniques on $c$-plane sapphire. 


\section{EXPERIMENTAL}

Different types of samples were investigated: bulk GaN, homoepitaxial GaN and heteroepitaxial GaN layers grown by MOCVD and MBE. The investigated GaN bulk crystals were grown at the High Pressure Research Center, Unipress, Warsaw by the hightemperature high-pressure method at $1800 \mathrm{~K}$ and $15 \mathrm{kbar}$ [5]. They have hexagonal platelet shape with lateral sizes of 3 to $4 \mathrm{~mm}$. One of the bulk crystals was $\mathrm{Mg}$-doped, the other was nominally undoped and overgrown by MOCVD with a $3 \mu \mathrm{m}$ thick undoped $\mathrm{GaN}$ layer [6]. For this, a substrate temperature of $1050^{\circ} \mathrm{C}$ was used similar to that applied for MOCVD of the two heteroepitaxial GaN layers deposited on $c$-plane sapphire. The growth conditions for these layers provided by S. DenBaars (University of California Santa Barbara) and S. Nakamura (Nichia Chemical Industries) can be found in ref. [7] and [8]. In addition, a $4.5 \mu \mathrm{m}$ thick GaN layer grown by MBE with an electron cyclotron plasma source on $c$-plane sapphire at a substrate temperature of $820^{\circ} \mathrm{C}$ was investigated. In contrast to the MOCVD layers, this layer was deposited directly onto the nitridated substrate without growing a low-temperature buffer before.

A high-resolution X-ray diffractometer Philips X'Pert MRD equipped with a $\mathrm{Cu}$ sealed anode, a four crystal monochromator and a triple crystal analyzer was used for the measurements. This diffractometer was extended by a continous flow X-ray cryostat of Oxford Instruments enabling temperature dependent measurements from 10 to $630 \mathrm{~K}$. The lattice parameters were calculated from the scattering angles directly measured by triple axis $2 \theta$ scans [9].

\section{RESULTS}

The progress in the growth of GaN bulk crystals over the last few years has resulted in crystal platelets with lateral extensions of up to $10 \mathrm{~mm}$ characterized by very narrow $\mathrm{X}$ ray diffraction profiles [5] which are a prerequisite for the accurate determination of lattice parameters [9]. However, highly resolved reciprocal space maps (RSMs) for such crystals as shown in figure 1 for an overgrown GaN substrate indicate that even the small GaN crystals obviously can consist of several macroscopic grains. Within the area of about $1 \mathrm{~mm}^{2}$ illuminated by the X-ray beam, we found typically two or three grains contributing to the scattered signal as indicated by corresponding sharp intensity maxima in the (00l) RSMs at different $q_{x}$ values. From the difference in $q_{x}$ the tilt of the crystallographic orientation between different grains can be calculated to be in the range of a few hundred arcseconds. Additionally, the grains are characterized sometimes by slightly different lattice parameters as it is indicated by the small shift of the intensity maxima $\mathrm{A}$ and $\mathrm{B}$ along $q_{z}$ in figure 1 . The changes in lattice parameter $c$ by $8 \cdot 10^{-4} \AA$ between different grains can be ascribed to different impurity concentrations pointing to a possible preferential accumulation of impurities in some grains. 

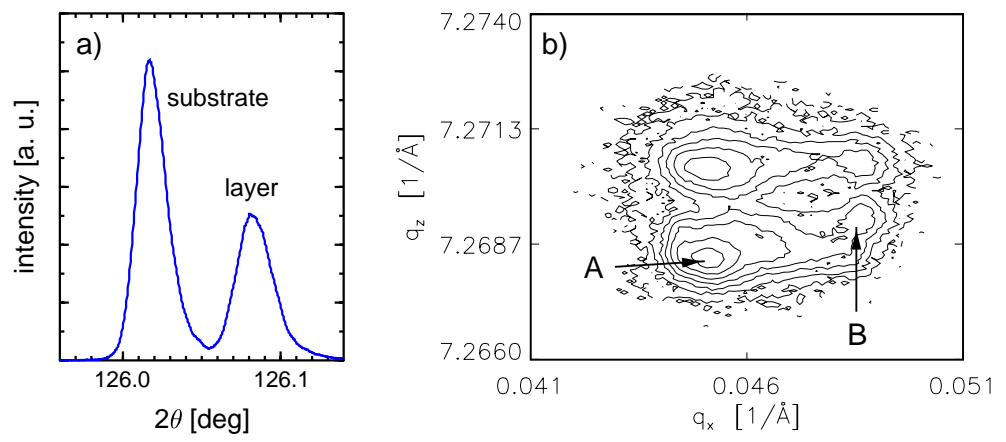

Figure 1. Triple axis $28 / \omega$ scan for the (006) reflection (a) and the corresponding RSM (b). The intensity maximums $A$ and $B$ are assigned to two grains of the substrate with different lattice parameter $c$.

The composition of the GaN bulk crystals of several grains with possibly slightly different lattice constants requires special efforts for an accurate determination of the temperature behavior of $\mathrm{GaN}$ lattice parameters. In our experiments, RSMs and triple axis $\omega$ scans were performed before measuring the scattering angle by $2 \theta / \omega$ scans. In this way it was ensured that the lattice parameters of the same grain were determined at all temperatures.

The RSM in figure 1(b) clearly demonstrates that the grain structure of the $\mathrm{GaN}$ substrate is reproduced in the overgrown layer as expected. The shift of substrate and layer intensity maxima along the $q_{z}$ axis corresponds to a difference in $c$ lattice parameter of $2 \cdot 10^{-3} \AA$. In earlier reports this behavior was ascribed to native defects or to different free electron concentrations in substrate and layer material [3]. RSMs of asymmetric reflections showed the pseudomorphic state of the layer in the whole temperature range investigated.

The lattice parameters of homoepitaxial layers and GaN bulk crystals come close to each other if the bulk material is $\mathrm{Mg}$ doped during growth [5]. This is confirmed by figure 2 where the lattice parameters $c$ of the intentionally undoped GaN bulk, the homoepitaxial layer deposited on it, and a $\mathrm{Mg}$ doped $\mathrm{GaN}$ crystal are plotted in dependence on the temperature.

Similarly to the GaN bulk and the homoepitaxial layer, several $\mu \mathrm{m}$ thick heteroepitaxial GaN layers on sapphire substrates were investigated. In figure 3 the results for the $c$ lattice parameters of layers grown by MOCVD and MBE are compared with those for the GaN bulk material and the homoepitaxial layer. For better clarity the latter data are represented as lines. Clear differences are visible for the two MOCVD samples. Whereas the sample MOCVD II has a $c$ lattice parameter which is clearly higher than that of the homoepitaxial layer in the whole temperature range, the $c$ values from the MOCVD I sample are near to those of the homoepitaxial layer. For the MBE grown layer the lattice parameters are between those of the MOCVD samples. Obviously, the slopes of the curves differ clearly for the different samples as well. 


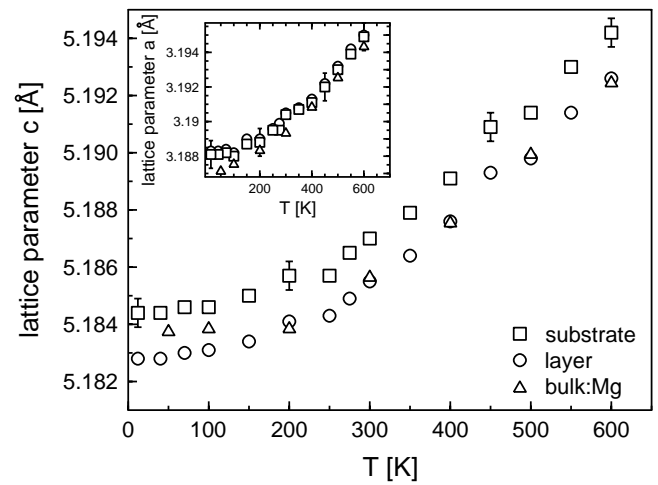

Figure 2. Lattice parameters $c$ in dependence on the temperature for an undoped bulk crystal (substrate) overgrown with a $3 \mu \mathrm{m}$ thick MOCVD layer and a Mg-doped GaN bulk crystal. The error bars are representative for all lattice parameters given. The lattice parameters a are shown in the inset.

\section{DISCUSSION}

From figure 2 the following informations can be obtained: (1) The temperature dependence of thermal expansion of $\mathrm{GaN}$ can be extracted. This has been done in ref. [10] assuming a linear expansion within each of the temperature ranges of 10 to $100 \mathrm{~K}, 100$ to $250 \mathrm{~K}$, and 250 to $600 \mathrm{~K}$. (2) Within the experimental errors, there is no indication for negative TEC at low temperatures. However, the amount of negative TEC as reported for other materials is often too small to be reflected in a significant change in lattice constants [11]. (3) The difference in lattice parameters between the homoepitaxial layer and the underlying substrate is constant over the whole investigated temperature range with an accuracy of $2 \cdot 10^{-4} \AA$.

The differences in $c$ lattice parameters for the heteroepitaxially grown layers can be partially attributed to different growth temperatures for the MOCVD and the MBE process. Since the growth temperature for the MOCVD samples is higher than that for the MBE sample, the thermal strain induced in the MOCVD layers should be higher. This is obviously the case for the MOCVD II sample for which the larger lattice parameters in growth direction indicate a stronger compressive biaxial strain. For the MOCVD I sample this model fails because the strain state seems to be the same as that of the MBE sample. A comparison of the $a$ lattice parameters (not shown here) indicates that this is probably due to large hydrostatic strain components in the MBE and MOCVD I sample.

The increase of the lattice parameters $c$ for all three heteroepitaxial layers is different from that of the bulk crystals and the homoepitaxial layer. Below $200 \mathrm{~K}$ the slope is close to zero as for the bulk and homoepitaxial GaN, but above $200 \mathrm{~K}$ the slope is clearly smaller than for the bulk material. This reflects directly the thermally induced 


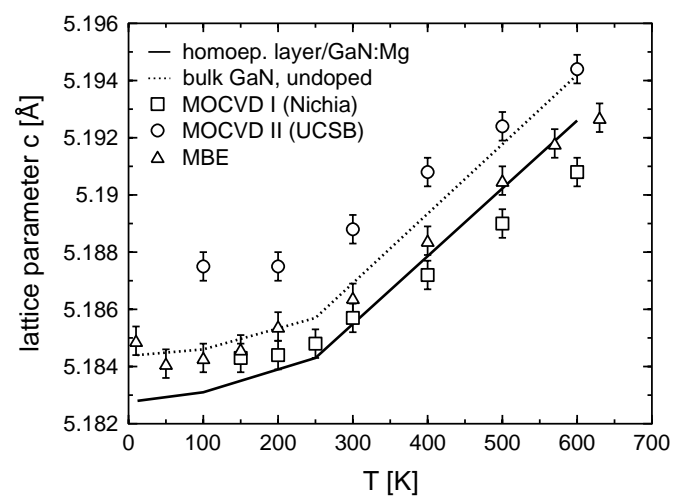

Figure 3. Comparison of the lattice parameters $c$ in dependence on the temperature for bulk GaN, homoepitaxial and different heteroepitaxial GaN layers.

strain in the epilayers grown on sapphire. On the other hand, the crossing of the curves for the heteroepitaxial layers with those for the bulk material far below the growth temperatures points to a strong influence of compressive hydrostatic strains in these layers.

\section{SUMMARY}

The temperature dependence of the lattice parameters of bulk, homoepitaxial and heteroepitaxial GaN was investigated and compared. Neither an influence of the electron concentration on the thermal expansion nor a negative thermal expansion at low temperatures was found in our experiments for bulk and homoepitaxial GaN. The comparison of results for heteroepitaxial samples with bulk values points to a superposition of thermally induced biaxial strain and compressive hydrostatic strain in the heteroepitaxial layers.

\section{ACKNOWLEDGEMENT}

The authors would like to thank I. Grzegory and S. Porowski (High Pressure Center, Unipress, Warsaw) for the growth of the GaN single crystals and P. Prystawko for the MOCVD growth of the GaN homoepitaxial layer. The heteroepitaxial MOCVD layers were generously provided by S. DenBaars (University of California Santa Barbara) and S. Nakamura (Nichia Chem. Ind.) which is kindly acknowledged.

\section{REFERENCES}

1. W. Rieger, T. Metzger, H. Angerer, R. Dimitrov, O. Ambacher and M. Stutzmann, Appl. Phys. Lett., 68, 970 (1996). 
2. W. Shan, R. J. Hauenstein, A. J. Fischer, J. J. Song, W. G. Perry, M. D. Bremser, R. F. Davis and B. Goldenberg, Mat. Res. Soc. Symp. Proc., 449, 841 (1997).

3. M. Leszczynski, J. Bak-Misiuk, J. Domagala and T. Suski, Mat. Res. Soc. Symp. Proc., 468, 311 (1997).

4. C. Kisielowski, J. Krüger, S. Ruvimov, T. Suski, J. W. Ager III, E. Jones, Z. Liliental-Weber, M. Rubin, E. R. Weber, M. D. Bremser and R. F. Davis, Phys. Rev. B, 54, 17745 (1996).

5. S. Porowski, J. Cryst. Growth, 189/190, 153 (1998).

6. J. L. Weyher, P. D. Brown, A. R. A. Zauner, S. Muller, C. B. Boothroyd, D. T. Foord, P. R. Hagemann, C. J. Humphreys, P. K. Larsen, I. Grzegory and S. Porowski, J. Cryst. Growth, 204, 419 (1999).

7. B. Heying, X. H. Wu, S. Keller, Y. Li, D. Kapolnek, B. P. Keller, S. P. DenBaars and J. S. Speck, Appl. Phys. Lett., 68, 643 (1996).

8. S. Nakamura, M. Senoh, N. Iwasa, S. Nagahama, T. Yamada and T. Mukai, Jpn. J. Appl. Phys., 34, L1332 (1995).

9. $\quad$ P. F. Fewster and N. L. Andrew, J. Appl. Cryst., 28, 451 (1995).

10. V. Kirchner, H. Heinke, D. Hommel, J. Z. Domagala and M. Leszczynski, submitted to Appl. Phys. Lett.

11. K. Wang and R. R. Reeber, Mat. Res. Soc. Symp. Proc., 482, 863 (1998). 